\title{
General approach for the description of size effects in ferroelectric nanosystems
}

\author{
Eugene A. Eliseev $\cdot$ Anna N. Morozovska
}

Received: 13 March 2009/Accepted: 6 April 2009/Published online: 23 April 2009

(c) The Author(s) 2009. This article is published with open access at Springerlink.com

\begin{abstract}
We propose general analytical approach for the description of size effect influence on polarization and dielectric susceptibility in ferroelectric nanosystems based on the two-parametric direct variational method and Landau-Ginzburg-Devonshire phenomenology. The essence of the approach is to solve Euler-Largange boundary problem for polarization distribution exactly in paraelectric phase without ferroelectric nonlinearity and then to use the linearized solution for derivation of the approximate analytical expression for spontaneous polarization distribution in ferroelectric phase with the average polarization and characteristic spatial scale as variational parameters. Corresponding polarization distributions calculated within the approach in thin ferroelectric films, nanowires and nanotubes were compared with the available exact solution of Landau-Ginzburg-Devonshire equation or approximate results obtained earlier from the one parametric solution. Perfect agreement between the exact solution and obtained approximate ones is demonstrated. The realization of the proposed scheme of the two-parametric direct variational method seems even simpler than the one-parametric scheme based on the Landau-Ginzburg-Devonshire free energy expansion with renormalized coefficients, while the validity range of two-parametric solution is much wider and the accuracy is higher. So, obtained analytical results have methodological importance for calculation of the
\end{abstract}

\section{E. A. Eliseev}

Institute for Problems of Materials Science, NAS of Ukraine,

Krjijanovskogo 3, 03142 Kiev, Ukraine

A. N. Morozovska ( $\square)$

V. Lashkarev Institute of Semiconductor Physics,

NAS of Ukraine, 41, pr. Nauki, 03028 Kiev, Ukraine

e-mail: morozo@i.com.ua phase diagram size effects, polarization distribution, all related polar, dielectric, piezoelectric and pyroelectric properties of single-domain ferroelectric nanoparticles and thin films. The proposed method is applicable to different ferroic nanosystems.

\section{Introduction}

Ferroelectric nanosystems open the way to obtain a variety of new unique electro-mechanical, electronic and dielectric properties, a lot of which are useful for applications, such as ferroelectric memories, the ability to store and release energy in well-regulated manners, making them very useful for sensors and actuators, compact electronics, pyrosensors and thermal imaging [1-3].

The substantial progress in synthesis of various ferroelectrics nanosystems, like epitaxial films [4], nanoparticles with controllable sizes [5], arrays of tubes and rods [6-9], the local characterization of their polar properties [10-12] and domain structure [13], triggered the renovation of interest to ferroic nanosystems theoretical description. It is worth to note the enormous achievements of both the phenomenological [14] and microscopic [15] theories, their recent advances in different fields like the description of nanorods $[16,17]$, size effects in thin films [18, 19], ferroelectric nanoparticles [20-22]; flexoelectric effect influence on the intrinsic properties [23, 24] and response [25-27] of the nanosystems; the developed analytical model accounting for depolarization field as well as the formation of misfit dislocations [28-30]. However, despite this progress, the phenomenological theory lacks a general method, suitable for the solution of vast variety of different problems of ferroics description. 
The possibility to govern the appearance of phase transitions at any arbitrary temperature has been demonstrated in nanosized materials due to the so-called size-driven phase transition. Such transitions were observed in many solids, including ferroelectric, ferromagnetic and ferroelastic ones [31]. For instance, it is generally accepted, that the ferroelectric properties disappear when the particle size decreases below the critical one $[1,32,33]$. Actually, it is well known that depolarization electric fields exist in the majority of confined ferroelectric systems [34] and causes the size-induced ferroelectricity disappearance in thin films and spherical particles [35, 36].

The phenomenological description of ferroelectricity in spatially confined systems based on the one-parametric direct variational method applied to the Landau-GinzburgDevonshire (LGD) free energy functional has been recently proposed [37-39]. For instance, we proved that the reason of the polar properties enhancement and conservation in ferroelectric nanorods is the stress coupled with polarization via electrostriction effect under the strong decrease of depolarization field with particle length increase. Briefly, the scheme based on one-parametric direct variational method is the following.

- Firstly the analytical solution of the linearized EulerLagrange boundary problem obtained from the LGD free energy functional minimization is derived. This solution corresponds to the polarization distribution in the paraelectric phase of the system, where the nonlinearity can be neglected in the weak external electric field. The average paraelectric susceptibility diverges in the point where the paraelectric phase loses its stability, so corresponding expression for the transition temperature $T_{c r}$ could be found directly from the condition of zero inverse susceptibility.

- In order to study the system behavior in ferroelectric phase, the coordinate-dependent part of the paraelectric solution is chosen as the trial one with its amplitude as variational parameter. After the integration of LGD free energy functional over the particle volume with the trial function we obtained the renormalized free energy with expansion coefficients depending on temperature $T$ and the particle sizes. The polarization amplitude can be determined from the algebraic equation obtained after the minimization of the renormalized free energy. If the analytical (exact or approximate) integration is possible it leads to the corresponding analytical expressions for renormalized coefficients size dependences.

The main advantage of the one-parametric direct variational method is the principal possibility to obtain analytical results, while the typical disadvantage is lengthy integration of the terms in LGD functional in order to obtain renormalized coefficients.

In the paper we propose general analytical approach for the description of size effect of polarization and dielectric susceptibility in ferroelectric nanosystems based on selfconsistent method of successive approximations. Here the first step is to find the deviation of polarization distribution from its average value. The amplitude and spatial scale of distribution appear to be dependent on average polarization due to the system nonlinearity. Next step is to look for the average value of polarization from the full distribution allowing for deviation in a self-consistent manner. Mathematically this method is equivalent to the two-parametric direct variational method with the average polarization and the distribution length scale as variational parameters. However the proposed scheme is free from the complex integration of the LGD free energy expansion coefficients, instead we solved the one transcendental equation for average polarization determination. Corresponding polarization distributions calculated within the approach in thin ferroelectric films, nanowires and nanotubes were compared with the available exact solution of LGD-equation or approximate results obtained earlier from the one-parametric solution applied to the LGD free energy.

\section{General approach}

For perovskite (cubic) symmetry the free energy expansion on the powers of polarization $P_{3}$ and strain $u_{i j}$ has the form:

$F=$

$$
\begin{aligned}
& \int_{V} d^{3} r\left(\frac{a_{1}^{u}(T)}{2} P_{3}^{2}+\frac{a_{11}^{u}}{4} P_{3}^{4}+\frac{a_{111}}{6} P_{3}^{6}\right. \\
& \left.+\frac{g}{2}\left(\nabla P_{3}\right)^{2}-P_{3}\left(E_{0}+\frac{E_{3}^{d}}{2}\right)-q_{i j 33} u_{i j} P_{3}^{2}+\frac{c_{i j k l}}{2} u_{i j} u_{k l}\right) \\
& +\int_{S} d^{2} r\left(\frac{a_{S}}{2} P_{3}^{2}+\frac{\beta_{S}}{4} P_{3}^{4}\right) .
\end{aligned}
$$

Coefficient $a_{1}^{u}(T)$ explicitly depends on temperature $T$. Coefficients $a_{S}, a_{111}$ are supposed to be temperature independent, positive constant $g$ determines magnitude of the gradient energy. $E_{3}^{d}$ is the depolarization field (if any). Tensor $c_{i j k l}$ is positively defined, also $a_{111}>0$. Free energy (1) is minimal when the system temperature and volume are fixed (i.e. strain components are defined at the nanostructure boundaries).

Below we consider the case of 1D-polarization distributions corresponding to mono-domain systems. The minimization of the free energy on polarization and strain components gives the following equations of state 
$\left(a_{1}^{u}-q_{i j 33} u_{i j}\right) P_{3}+a_{11}^{u} P_{3}^{3}+a_{111} P_{3}^{5}-g \frac{\partial^{2} P_{3}}{\partial x_{k} \partial x_{k}}=E_{0}+E_{3}^{d}$,

$-q_{i j 33} P_{3}^{2}+c_{i j k l} u_{k l}=\sigma_{i j}$.

These equations should be supplemented with Maxwell equations for electrostatic electric field and compatibility conditions for strain and equilibrium conditions for stress components [40].

In some cases elastic sub-problem could be solved at fixed polarization (see details in Appendix 1). Results are summarized in Table 1 for mechanically free system $\left(\sigma_{i j}=0\right)$, clamped system $\left(u_{i j}=0\right)$ and "mixed" case corresponding to thin epitaxial films on thick and/or rigid substrate [41]. In the latter case $u_{m}$ is the misfit strain in plane of the system due to the mechanical incompatibility between the film and substrate.

Shear strain components are zero in these cases, $u_{12}=$ $u_{13}=u_{23}=0$. It should be noted, that the solutions listed in Table 1 are valid only for the polarization distributions, specified in the second column of the table. In the case of arbitrary distribution of polarization either compatibility or equilibrium conditions could be not satisfied for the elastic fields from Table 1. For instance, in the case of onedimensional distribution of polarization, $P_{3}\left(x_{1}\right)$, in the elastically free system components $u_{22}, u_{33}$ from second row should be replaced with their mean values. One of the consequences of such distribution is the stress localization in the vicinity of domain walls (see e.g. papers of Cao and Cross [42] and Zhirnov [43]). It should be noted, that the influence of the misfit dislocation on the misfit strain $u_{m}$ relaxation could be taken into account by the renormalization of $u_{m}$ (see e.g. Speck and Pompe paper [44]).

After substitution of to the strain field into equation of state (2a) one could get the renormalized expansion coefficients, presented in Table 2.

\section{Polarization distribution in ferroelectric films}

For the case of ferroelectric film with thickness $L$, occupying the region $-L / 2<x \leq L / 2$, the depolarization field $E_{3}^{d}$ is absent, if the polarization vector $P_{3}(x)$ is laying in the film plane. Taking into account the renormalization listed in the Table 2, one could rewrite Eq. 2a as

$\left\{\begin{array}{l}\alpha P_{3}+\beta P_{3}^{3}-g \frac{\partial^{2} P_{3}}{\partial x^{2}}=E_{0} \\ \left.\left(P_{3} \pm \lambda \frac{d P_{3}}{d x}\right)\right|_{x= \pm L / 2}=0, \quad \lambda=\frac{g}{a_{S}},\end{array}\right.$

Here we introduced extrapolation length $\lambda$ in boundary condition. Below we suppose standard dependence on temperature, $\alpha=\alpha_{T}\left(T-T_{C}\right)$, though critical temperature $T_{C}$ could be different from bulk value due to the influence of misfit strain (see Table 2).

In the absence of electric field Eq. 3 has an exact solution (see e.g. Refs. [45-47]):

$P_{3}(x)=P_{b} \sqrt{\frac{2 m}{1+m}} s n\left(\frac{x}{R_{c} \sqrt{1+m}}+K(m) \mid m\right)$

Here $P_{b}=\sqrt{-\alpha / \beta}$ and $R_{c}=\sqrt{g /(-\alpha)}$ are the spontaneous polarization and the correlation radius of bulk material at $T \leq T_{\mathrm{c}}, \operatorname{sn}(u \mid m)$ is the elliptical sine function [48]. Constant $m$ should be determined from the boundary conditions, which gives the following equation:

$L=2 R_{c} \sqrt{1+m}(K(m)-F(\arcsin (\varphi(m, \lambda)), m))$

$\varphi(m, \lambda)$

$=\sqrt{\frac{1+m}{2 m}\left(1+\left(\frac{R_{c}}{\lambda}\right)^{2}-\sqrt{\left(1+\left(\frac{R_{c}}{\lambda}\right)^{2}\right)^{2}-\frac{4 m}{(1+m)^{2}}}\right)}$

Here $\mathrm{K}(m)$ and $\mathrm{F}(\varphi, m)$ are complete and incomplete elliptic integrals of the first kind respectively. Distributions of spontaneous polarization in films of different thickness for different values of extrapolation length are shown in Fig. 1a, b, where $R_{c}^{0}=\sqrt{g /\left(\alpha_{T} T_{C}\right)}$ is the bulk correlation radius at $T=0$.

At $m \rightarrow 0$ polarization (4) disappears, which means transition from ferroelectric to paraelectric phase. In this limit relation $(5 \mathrm{a}, 5 \mathrm{~b})$ is reduced to the condition of phase transition in thin films:

$\tan \left(\frac{L}{2 R_{c}}\right)=\frac{R_{c}}{\lambda}$.
Table 1 Strain field in ferroelectric systems

\begin{tabular}{lllll}
\hline & $\begin{array}{l}\text { Polarization } \\
\text { distribution }\end{array}$ & \multicolumn{4}{l}{ Non-trivial strain components } \\
\cline { 3 - 6 } & $P_{3}=$ const & $\frac{\left(-c_{12} q_{11}+c_{11} q_{12}\right) P_{3}^{2}}{\left(c_{11}+2 c_{12}\right)\left(c_{11}-c_{12}\right)}$ & & $u_{22}$ \\
\hline Free system & $P_{3}=$ const & 0 & 0 & $\frac{\left(\left(c_{11}+c_{12}\right) q_{11}-2 c_{12} q_{12}\right) P_{3}^{2}}{\left(c_{11}+2 c_{12}\right)\left(c_{11}-c_{12}\right)}$ \\
Clamped system & $P_{3}\left(x_{3}\right)$ & $u_{m}$ & $u_{m}$ & $\frac{q_{11}}{c_{11}} P_{3}^{2}-\frac{2 c_{12}}{c_{11}} u_{m}$ \\
Films with out of plane $\mathbf{P}$ & $P_{3}\left(x_{1}\right)$ & $\frac{q_{12}}{c_{11}} P_{3}^{2}-\frac{2 c_{12}}{c_{11}} u_{m}$ & $u_{m}$ & $u_{m}$ \\
Films with in plane $\mathbf{P}$ & & & & \\
\hline
\end{tabular}


Table 2 Free energy expansion coefficients renormalization

\begin{tabular}{lll}
\hline & $\alpha$ & $\beta$ \\
\hline Free system & $a_{1}^{u}$ & $a_{11}^{u}-4 \frac{\left(q_{11}-q_{12}\right)^{2}}{3\left(c_{11}-c_{12}\right)}-2 \frac{\left(q_{11}+2 q_{12}\right)^{2}}{3\left(c_{11}+2 c_{12}\right)}$ \\
Clamped system & $a_{1}^{u}$ & $a_{11}^{u}$ \\
Films with out of plane $\mathbf{P}$ & $a_{1}^{u}-2 q_{12} u_{m}+q_{11} \frac{2 c_{12}}{c_{11}} u_{m}$ & $a_{11}^{u}-\frac{q_{11}^{2}}{c_{11}}$ \\
Films with in plane $\mathbf{P}$ & $a_{1}^{u}-\left(q_{11}+q_{12}\right) u_{m}+q_{12} \frac{2 c_{12}}{c_{11}} u_{m}$ & $a_{11}^{u}-\frac{q_{12}^{2}}{c_{11}}$ \\
\hline
\end{tabular}

Fig. 1 a, b Polarization distribution inside the films with extrapolation length $\lambda / R_{c}=0$ (a) and 1.5 (b). Solid curves were plotted from exact distribution (4), dotted curves were plotted from approximate definitions (8-9). Film thickness values are $L / R_{c}=3.5,5,10,20$ (curves 1-4 in plot (a)) and $L / R_{c}=2.5,5,10,20$ (curves 1-4 in plot (b)). c Critical temperature dependence on the inverse film thickness for $\lambda / R_{c}^{0}=0,1,2,3$ (curves 1-4). Solid curves were plotted from Eq. 4, dotted curves were plotted from approximate definitions (10-11). d Average polarization dependence on the inverse film thickness for $\lambda / R_{c}=0,1.5,3$ (curves $1-3$ ). Solid curves correspond to exact expressions, dashed and dotted curves were plotted from approximate definitions (9) and (10) correspondingly
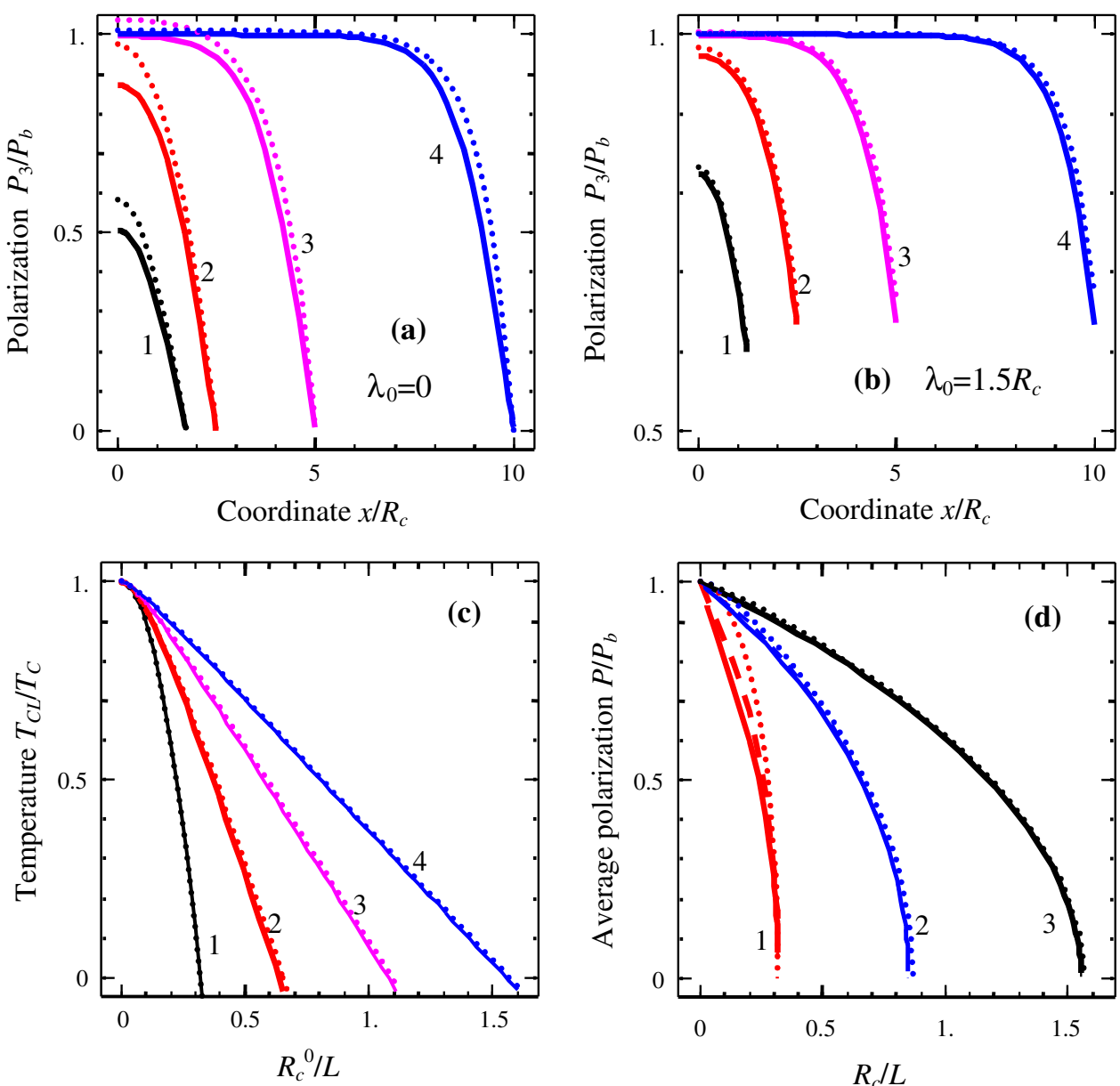

At fixed temperature Eq. 6a determines the critical thickness $L_{c r}=2 R_{c} \arctan \left(R_{c} / \lambda\right)$ of ferroelectric films, i.e. in the film with thickness $L<L_{c r}$ ferroelectric phase is unstable. At fixed thickness Eq. 6a determines the critical temperature of transition (see Fig. 1c). The following approximation for the transition temperature were found (see Appendix 3):

$T_{C L} \approx T_{C}-\frac{2 \pi^{2} g / \alpha_{T}}{\pi^{2} L \lambda+2 L^{2}}$

Note that relationship (6b) is exact in two limits of low and high extrapolation length values. The comparison of exact (6a) and approximate (6b) expressions for critical temperature is shown in Fig. 1c (see solid and dotted curves respectively). It is seen that approximation (6b) is very close to exact dependence.

The exact solution (4) involves higher transcendental function and is limited to the cases of ferroelectric phase and zero external electric field. The solutions for the first derivatives (susceptibility and pyroelectric coefficient) are also available [47], but they have even more sophisticated structure. These lead us to attempt to find the approximate solution of Eq. 3 in terms of elementary functions, valid in both paraelectric and ferroelectric phase.

Let us look for the solution in the form $P_{3}(x)=$ $\bar{P}+p(x)$, where $\bar{P}=\int_{-L / 2}^{L / 2} P_{3}(x) d x / L$ is the averaged polarization, $p$ is the deviation, regarded small in 
ferroelectric phase: $|p(x)| \ll|\bar{P}|$. So, linearized problem (3) acquires the form:

$$
\left\{\begin{array}{l}
\left(\alpha+3 \beta \bar{P}^{2}\right) p-g \frac{\partial^{2} p}{\partial x^{2}}=E_{0}-\left(\alpha \bar{P}+\beta \bar{P}^{3}\right), \\
\left.\left(p \pm \lambda \frac{d p}{d x}\right)\right|_{x= \pm L / 2}=-\bar{P}
\end{array}\right.
$$

The solution of the linear problem (7) is the following:

$P_{3}(x)=\left(\frac{E_{0}+2 \beta \bar{P}^{3}}{\alpha+3 \beta \bar{P}^{2}}\right) f\left(x, L, \sqrt{\frac{g}{\alpha+3 \beta \bar{P}^{2}}}\right)$,

$f\left(x, L, R_{0}\right)=1-\frac{\cosh \left(x / R_{0}\right)}{\cosh \left(L / 2 R_{0}\right)+\left(\lambda / R_{0}\right) \sinh \left(L / 2 R_{0}\right)}$,

$R_{0}=\sqrt{\frac{g}{\alpha+3 \beta \bar{P}^{2}}}$.

Here $R_{0}$ is the characteristic length scale that should be determined self-consistently. The average polarization should be determined self-consistently from the spatial averaging Eqs. $8 \mathrm{a}$ and $8 \mathrm{~b}$ as

$\bar{P}=\left(\frac{E_{0}+2 \beta \bar{P}^{3}}{\alpha+3 \beta \bar{P}^{2}}\right) \bar{f}\left(L, \sqrt{\frac{g}{\alpha+3 \beta \bar{P}^{2}}}\right)$.

$\bar{f}\left(L, R_{0}\right)=1-\frac{\sinh \left(L /\left(2 R_{0}\right)\right) 2 R_{0} / L}{\cosh \left(L /\left(2 R_{0}\right)\right)+\left(\lambda / R_{0}\right) \sinh \left(L /\left(2 R_{0}\right)\right)}$

Allowing for Eqs. 9a and 8a could be rewritten as $P_{3}(x)=\bar{P} f\left(x, L, R_{0}\right) / \bar{f}\left(L, R_{0}\right)$.

In fact, Eq. 9 is the transcendental equation for the average polarization determination. But in contrast to Eq. 5 it involves only elementary functions. Approximation for average polarization at $E_{0}=0$ could be obtained from the expression of bulk spontaneous polarization $P_{b}=$ $\sqrt{\alpha_{T}\left(T_{C}-T\right) / \beta}$ by substitution of $T_{C}$ with $T_{C L}$ :

$\bar{P} \approx \sqrt{\frac{\alpha_{T}\left(T_{C L}-T\right)}{\beta}}$

Approximate distributions of polarization $(8 \mathrm{a}, 8 \mathrm{~b})$ are shown in Fig. 1a, b in comparison with exact expressions (4). It is seen that even for the most "problematic" case $\lambda=0$ corresponding to the maximal deviation of polarization from the average value the approximate profiles ( $8 \mathrm{a}$, $8 b)$ give quantitative description of exact ones. For the average polarization dependence on the film thickness (see Fig. 1d) the approximate dependences are even closer to exact ones for the most values of extrapolation length.

The proposed approach to the confined ferroelectric system description is analogous to direct variational method with two variational parameters, namely the average polarization $\bar{P}$ and the characteristic length scale $R_{0}$. The dependence of the latter on the average polarization reflects changes of the polarization distribution when approaching the phase transition point, which is the feature, present in the exact solution (4) via parameter $m$, changing from 0 to 1 .

If the polarization is pointed perpendicular to the film surface and the depolarization field is present in the system $[49,50]$ the proposed method gives essentially the same results as the one parametric variational method did (see Appendix 2), since in this case the characteristic length scale appeared to be practically independent on temperature and film thickness and is determined solely by the depolarization field screening [47].

The advantages of the developed approximate method and its high accuracy encourage one to apply this method for other ferroic system of different geometry where exact solutions are not available.

\section{Polarization distribution in ferroelectric nanowires}

In contrast to the thin films on the substrate, the elastic field of the spontaneous strain $u_{i j} \sim P_{3}^{2}$ inside cylindrical ferroelectric nanoparticles is rather complicated because of the polarization distribution, which leads to the appearance of non-local terms, involving the term with polarization mean square value $P_{3} \overline{P_{3}^{2}}$ in the polarization equation of state [51]. The proposed method allows taking into account these terms by involving additional parameter $\overline{P_{3}^{2}}$, which makes the consideration very cumbersome. At the same time, one could get the quantitatively correct picture of the size effect in ferroelectric nanowires neglecting the distinction of strain field from the one of bulk system (see Table 1). Here we suggest using renormalized expansion coefficients for free system from Table 2 as an approximation for real system.

Considering long cylindrical nanoparticles (nanowires, long nanorods etc.) one could neglect the effects of depolarization field and faces of particles. Under such conditions, equation of state (2a) should be rewritten as:

$\left\{\begin{array}{l}\alpha P_{3}-g\left(\frac{\partial^{2}}{\partial \rho^{2}}+\frac{1}{\rho} \frac{\partial}{\partial \rho}\right) P_{3}+\beta P_{3}^{3}=E_{0}, \\ \left.\left(P_{3}+\lambda \frac{d P_{3}}{d \rho}\right)\right|_{\rho=R}=0, \quad \lambda=\frac{g}{a_{S}}, \\ \left|P_{3}(\rho=0)\right|<\infty\end{array}\right.$

Let us find the solution valid in both paraelectric and ferroelectric phase in the form $P_{3}(\rho)=\bar{P}+p(\rho)$, where $\bar{P}=\frac{2}{R^{2}} \int_{0}^{R} P_{3}(\rho) \rho d \rho$ is the averaged polarization, $p$ is the deviation, regarded small in ferroelectric phase: $|p(\rho)| \ll$ $|\bar{P}|$. So, linearized problem (11) acquires the form:

$\left\{\begin{array}{l}\left(\alpha+3 \beta \bar{P}^{2}\right) p-g\left(\frac{\partial^{2}}{\partial \rho^{2}}+\frac{1}{\rho} \frac{\partial}{\partial \rho}\right) p=E_{0}-\left(\alpha \bar{P}+\beta \bar{P}^{3}\right), \\ \left.\left(p+\lambda \frac{\partial p}{\partial \rho}\right)\right|_{\rho=R}=-\bar{P},|p(\rho=0)|<\infty\end{array}\right.$ 
The solution of the problem has the form:

$P_{3}(\rho)=\left(\frac{E_{0}+2 \beta \bar{P}^{3}}{\alpha+3 \beta \bar{P}^{2}}\right) c\left(\rho, R, R_{0}\right)$,

$c\left(\rho, R, R_{0}\right)=1-\frac{I_{0}\left(\rho / R_{0}\right)}{I_{0}\left(R / R_{0}\right)+\left(\lambda / R_{0}\right) I_{1}\left(R / R_{0}\right)}$.

Here $R_{0}=\sqrt{g /\left(\alpha+3 \beta \bar{P}^{2}\right)}, I_{0}(x)$ and $I_{1}(x)$ are modified Bessel functions of the zero and first orders respectively ${ }^{1}$. The average polarization should be determined selfconsistently from the spatial averaging in Eq. 13 as

$\bar{P}=\left(\frac{E_{0}+2 \beta \bar{P}^{3}}{\alpha+3 \beta \bar{P}^{2}}\right) \bar{c}\left(R, R_{0}\right)$.

$\bar{c}\left(R, R_{0}\right)=1-\frac{2 I_{1}\left(R / R_{0}\right)\left(R_{0} / R\right)}{I_{0}\left(R / R_{0}\right)+\left(\lambda / R_{0}\right) I_{1}\left(R / R_{0}\right)}$

Allowing for Eqs. 14a and 13a could be rewritten as:

$P_{3}(\rho)=\bar{P}\left(E_{0}\right) \frac{c\left(\rho, R, R_{0}\right)}{\bar{c}\left(R, R_{0}\right)}$

Using Eqs. (13a, 13b)-(14a, 14b), one could calculate dielectric susceptibility $\chi(\rho)=\partial P_{3}(\rho) / \partial E_{0}$ and its mean value $\bar{\chi}=\partial \bar{P} / \partial E_{0}$. Using the relation $\partial R_{0} / \partial \bar{P}=$ $-R_{0} 3 \beta \bar{P} /\left(\alpha+3 \beta \bar{P}^{2}\right)$, differentiation of Eq. 14a gives:

$$
\begin{aligned}
\bar{\chi}= & \frac{\bar{c}\left(R, R_{0}\right)}{\alpha+3 \beta \bar{P}^{2}} \\
& \times\left(1-\frac{6 \beta \bar{P}\left(\alpha \bar{P}+\beta \bar{P}^{3}-E_{0}\right)}{\left(\alpha+3 \beta \bar{P}^{2}\right)^{2}} \bar{c}\left(R, R_{0}\right)\right. \\
& \left.+\frac{3 \beta \bar{P}\left(E_{0}+2 \beta \bar{P}^{3}\right)}{\left(\alpha+3 \beta \bar{P}^{2}\right)^{2}} \frac{\partial \bar{c}\left(R, R_{0}\right)}{\partial R_{0}} R_{0}\right)^{-1}
\end{aligned}
$$

Here the derivative has the view:

$$
\begin{aligned}
& \frac{\partial \bar{c}\left(R, R_{0}\right)}{\partial R_{0}} R_{0} \\
& =\frac{2 R_{0}^{2}\left(R I_{0}\left(R / R_{0}\right)^{2}-(R+2 \lambda) I_{1}\left(R / R_{0}\right)^{2}-2 R_{0} I_{0}\left(R / R_{0}\right) I_{1}\left(R / R_{0}\right)\right)}{R\left(R_{0} I_{0}\left(R / R_{0}\right)+\lambda I_{1}\left(R / R_{0}\right)\right)^{2}} .
\end{aligned}
$$

Similarly to (16a, 16b), one could obtain from Eqs. 13a and $13 \mathrm{~b}$ the susceptibility distribution:

\footnotetext{
1 The linearized solution for the polarization distribution in paraelectric phase and the averaged polarization was derived earlier in Refs. [32, 35] as $P_{3}(\rho)=\frac{E_{0}}{\alpha}\left(1-\frac{J_{0}\left(\rho / R_{c}\right)}{J_{0}\left(R / R_{c}\right)-\left(\lambda / R_{c}\right) J_{1}\left(R / R_{c}\right)}\right)$, where $R_{c}=$ $\sqrt{-g / \alpha}$. However the solution is invalid in ferroelectric phase, since the scale $R_{\mathrm{c}}$ is different from $R_{0}$ introduced in Eq. 4.
}

$$
\begin{aligned}
\chi(\rho)= & \left(\frac{1}{\alpha+3 \beta \bar{P}^{2}}+\frac{6 \beta \bar{P}\left(\alpha \bar{P}+\beta \bar{P}^{3}-E_{0}\right)}{\left(\alpha+3 \beta \bar{P}^{2}\right)^{2}}\right) c\left(\rho, R, R_{0}\right) \\
& -\bar{\chi} \frac{3 \beta \bar{P}\left(E_{0}+2 \beta \bar{P}^{3}\right)}{\left(\alpha+3 \beta \bar{P}^{2}\right)^{2}} \frac{\partial c\left(\rho, R, R_{0}\right)}{\partial R_{0}} R_{0}
\end{aligned}
$$

Here the derivative has the view:

$$
\begin{aligned}
\frac{\partial c\left(\rho, R, R_{0}\right)}{\partial R_{0}} R_{0}= & \frac{\rho I_{1}\left(\rho / R_{0}\right)}{R_{c} I_{0}\left(R / R_{0}\right)+\lambda I_{1}\left(R / R_{0}\right)} \\
& -\frac{R I_{0}\left(\rho / R_{0}\right)\left(\lambda I_{0}\left(R / R_{0}\right)+R_{0} I_{1}\left(R / R_{0}\right)\right)}{\left(R_{0} I_{0}\left(R / R_{0}\right)+\lambda I_{1}\left(R / R_{0}\right)\right)^{2}}
\end{aligned}
$$

The results of susceptibility and polarization distributions calculations are presented in Fig. 2 for different values of extrapolation length and nanowires radius. Dependence of extrapolation length on the rod radius proposed in Ref. [32] was ignored for the sake of simplicity.

Susceptibility is renormalized on the value $\chi_{b}=$ $-1 / 2 a_{1}(T)$, which is dielectric susceptibility of the bulk material. The drop of polarization and the increase of susceptibility in the vicinity of size-driven phase transition is obvious. Also the maximum of susceptibility near the surface of thick wires could be related to the decrease of polarization in this region. The similar effect was predicted for the ferroelectric films with in-plane polarization (i.e. without depolarization field) on the basis of exact solution [47].

In paraelectric phase higher order power terms of $\bar{P}$ could be neglected since $\bar{P}\left(E_{0} \rightarrow 0\right) \sim E_{0}$, so the transcendental Eq. 14a for the determination of $\bar{P}$ reduces to definition of susceptibility, $\bar{P}=E_{0} \bar{c}(R, \sqrt{g / \alpha(T)}) / \alpha$. Thus critical point of transition between paraelectric and ferroelectric phases corresponds to the zero denominator in Eq. 14b. At $\alpha(T)<0$ this condition can be rewritten in the form:

$J_{0}\left(R \sqrt{-\frac{\alpha\left(T_{C R}\right)}{g}}\right)-\lambda \sqrt{-\frac{\alpha\left(T_{C R}\right)}{g}} J_{1}\left(R \sqrt{-\frac{\alpha\left(T_{C R}\right)}{g}}\right)=0$.

Here $J_{n}(x)$ is the Bessel function of the $n$-th order.

Pade approximations of the solution of Eq. 18a for transition temperature $T_{C R}$ could be written as [17]:

$T_{C R} \approx\left\{\begin{array}{l}T_{C}-\frac{2}{\alpha_{T}}\left(\frac{g}{R \lambda+2 R^{2} / k_{01}^{2}}\right), \quad \lambda>0, \\ T_{C}-\frac{2}{\alpha_{T}}\left(g \frac{2 \lambda-R}{2 R \lambda^{2}}\right), \quad \lambda<0 .\end{array}\right.$

where $k_{01}=2.408 \ldots$ is the smallest positive root of equation $J_{0}(k)=0$. 
Fig. 2 Susceptibility $(\mathbf{a}, \mathbf{c})$ and polarization $(\mathbf{b}, \mathbf{d})$ distribution inside the nanorods, for different values of extrapolation length $\lambda / R_{c}=2(\mathbf{a}, \mathbf{b}) 5(\mathbf{c}, \mathbf{d})$. Nanorod radius values $R /$ $R_{c}=1.35,1.6,2,3,5,7.5$ (curves 1-6 in panels $(\mathbf{a}, \mathbf{b})$ ) and $R / R_{c}=0.8,1,1.4,2,3,5$ (curves 1-6 in panels $(\mathbf{c}, \mathbf{d})$ )
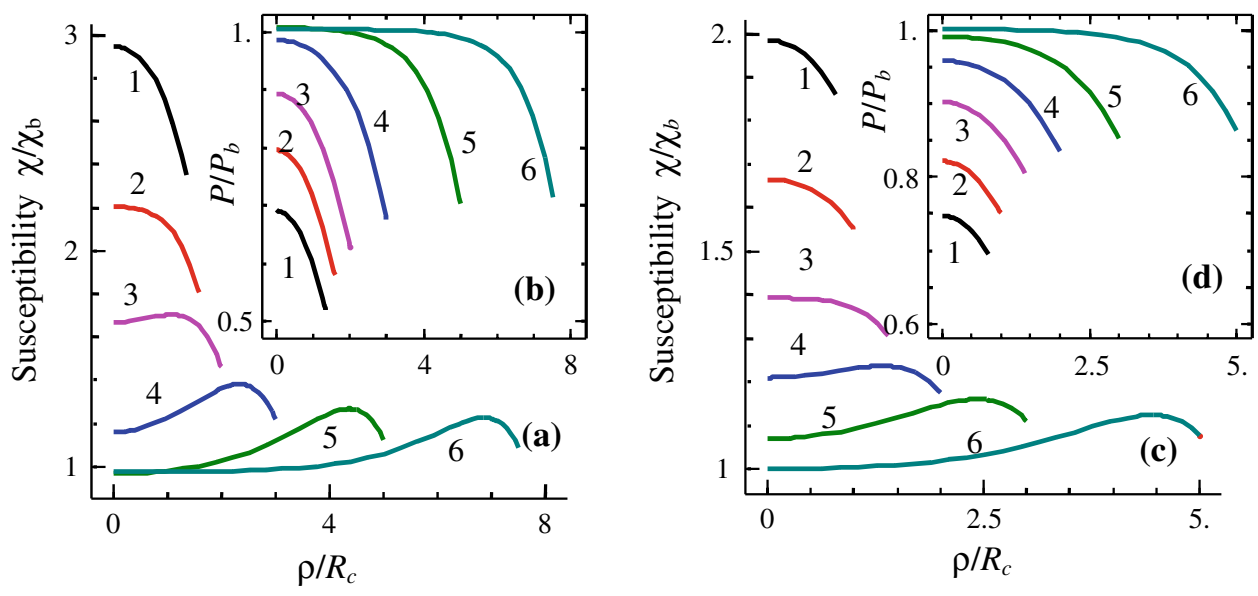

Fig. 3 a Critical temperature dependence on the inverse wire radius for $\lambda / R_{c}^{0}=0,1.5,3$ (curves 1-3). Solid curves represent the numerical solution of Eq. 18a, dotted curves are after approximate expression (18b). b Average polarization dependence on the inverse wire radius for $\lambda / R_{c}=0,1.5,3$ (curves 1-3). Solid and dashed curves correspond to approximate expressions (14a) and (19) respectively
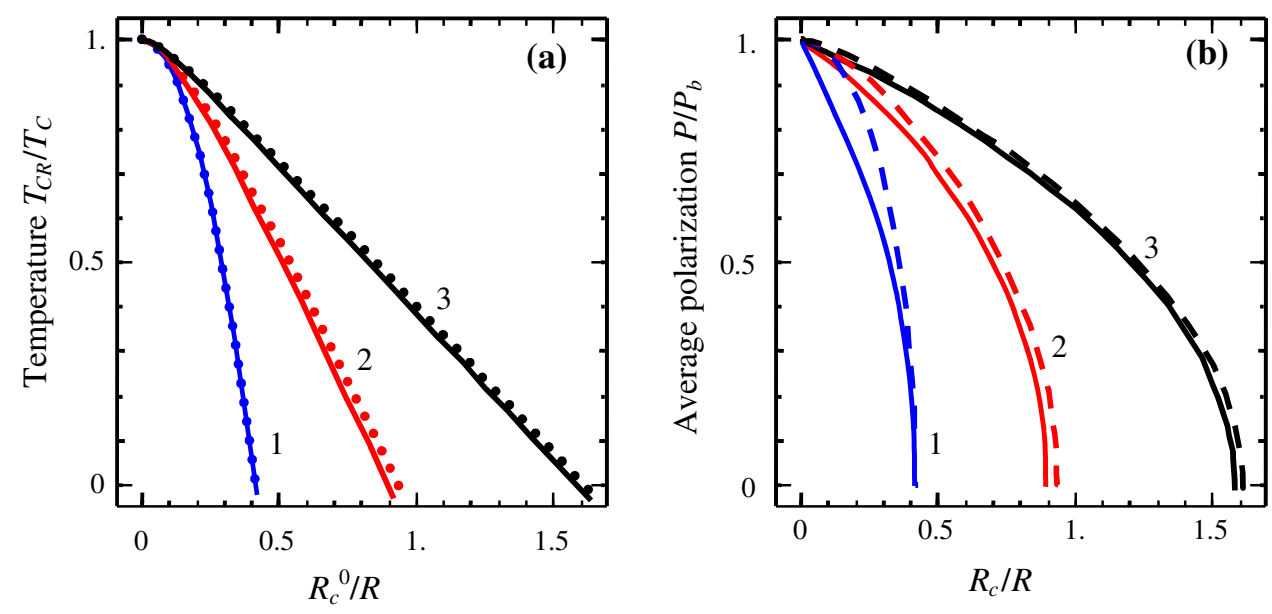

The comparison of the exact and approximate dependences is shown in Fig. 3a.

Similarly to the case of ferroelectric films with in plane polarization, the approximation for average spontaneous polarization at $E_{0}=0$ could be obtained from the expression of bulk spontaneous polarization $P_{b}=$ $\sqrt{\alpha_{T}\left(T_{C}-T\right) / \beta}$ by substitution of $T_{C}$ with $T_{C R}$, as it was widely used before [17, 37, 39]:

$\bar{P} \approx \sqrt{\frac{\alpha_{T}\left(T_{C R}-T\right)}{\beta}}$

The comparison of the different approximate expressions for the average polarization dependence on the rod radius is shown in Fig. 3b. It is seen that rough approximation (19) almost coincides with more rigorous expression for high values of extrapolation length.

It is obvious, that the results for nanorods could be generalized to the case of nanotubes of arbitrary sizes in straightforward way (see Appendix 4). The detailed analysis of the results for ferroelectric nanotubes will be presented elsewhere.

\section{Summary}

We propose general approach for the description of size effect of polarization distribution and transition temperature in ferroelectric nanosystems based on the two-parametric direct variational method and LGD phenomenology. The scheme of the method consists of three steps.

I. To obtain the Euler-Lagrange boundary problem for polarization distribution from the minimization of the LGD free energy functional.

II. To linearize the Euler-Lagrange boundary problem near the average value of polarization and to obtain the equation for deviation of polarization from its average value. The solution of this equation could be found by using standard methods and gives the polarization distribution with amplitude and length scale dependent on the average polarization.

III. To find the average polarization self-consistently by the averaging of the Euler-Lagrange equation solution, obtained on the step II and dependent on the average polarization. 
Allowing for the step III, the two-parametric scheme of the direct variational method is free from the complex integration in order to obtain the LGD free energy with renormalized expansion coefficients; instead we solved the only one transcendental equation for average polarization determination. Thus, the realization of the two-parametric scheme is simpler than the one-parametric scheme based on the LGD free energy expansion with renormalized coefficients, while we proved that the validity range of two-parametric solution is much wider and the accuracy is higher.

Obtained analytical results have priory methodological importance for calculation of the phase diagram size effects, polarization distribution, polar, dielectric, piezoelectric and pyroelectric properties of single-domain ferroelectric nanoparticles and thin films. The method is also applicable to different ferroic nanosystems.

\section{Appendix 1: elastic fields}

The equation of state (2b) should be supplemented with compatibility conditions for strain

$e_{i k l} e_{j m n} \partial^{2} u_{l n} / \partial x_{k} \partial x_{m}=0$

and equilibrium conditions for stress components

$\partial \sigma_{i j} / \partial x_{i}=0$.

Also boundary conditions should be specified.

For mechanically clamped system $\left(u_{k l}=0\right)$ internal elastic stress could be obtained from Eq. $2 \mathrm{~b}$ as $\sigma_{i j}=$ $-q_{i j 3} P_{3}^{2}$. It is stable only for homogeneous distribution of polarization, otherwise equilibrium condition (21) is not satisfied.

For mechanically free system $\left(\sigma_{i j}=0\right)$ nontrivial spontaneous strain components could be found from Eq. $2 \mathrm{~b}$ as

$$
\begin{aligned}
& u_{11}=u_{22}=\left(\frac{q_{11}+2 q_{12}}{c_{11}+2 c_{12}}-\frac{q_{11}-q_{12}}{c_{11}-c_{12}}\right) \frac{P_{3}^{2}}{3}, \\
& u_{33}=\left(\frac{q_{11}+2 q_{12}}{c_{11}+2 c_{12}}+2 \frac{q_{11}-q_{12}}{c_{11}-c_{12}}\right) \frac{P_{3}^{2}}{3} .
\end{aligned}
$$

Substitution of strain (22a) into Eq. 2a gives the following renormalization of coefficient before $P_{3}^{3}$ term:

$a_{11}^{\sigma}=a_{11}^{u}-4 \frac{\left(q_{11}-q_{12}\right)^{2}}{3\left(c_{11}-c_{12}\right)}-\frac{\left(q_{11}+2 q_{12}\right)^{2}}{3\left(c_{11}+2 c_{12}\right)}$,

while all the other coefficients remained the same.

The elastic fields in epitaxial ferroelectric film on thick and/or rigid substrate are found by Pertsev et al. [41]. In this case in plane components of strain should be fixed to the values, determined by the misfit, while normal components of stress should be zero.
For the polarization normal to film surface at $x_{3}=$ const the elastic fields are:

$$
\begin{aligned}
& \sigma_{12}=\sigma_{13}=\sigma_{23}=\sigma_{33}=0, \\
& \sigma_{11}=\sigma_{22}=\left(\frac{q_{11} c_{12}}{c_{11}}-q_{12}\right) P_{3}^{2}+\frac{u_{m}}{s_{11}+s_{12}}, \\
& u_{12}=u_{13}=u_{23}=0, u_{11}=u_{22}=u_{m}, \\
& u_{33}=\frac{q_{11}}{c_{11}} P_{3}^{2}-\frac{2 c_{12}}{c_{11}} u_{m}
\end{aligned}
$$

Here $u_{m}$ is the misfit strain in plane of the system due to the mechanical incompatibility between the film and substrate. Here we denote $\left(s_{11}+s_{12}\right)^{-1} \equiv c_{11}+c_{12}-2 c_{12}^{2} / c_{11}$.

Due to the strain (23b) expansion coefficients in (1) and (2a) should be renormalized as

$a_{1}^{m}=a_{1}^{u}-2 q_{12} u_{m}+q_{11} \frac{2 c_{12}}{c_{11}} u_{m}, a_{11}^{m}=a_{11}^{u}-\frac{q_{11}^{2}}{c_{11}}$

For the in-plane polarization (film plane $x_{1}=$ const) the elastic fields are:

$$
\begin{aligned}
& \sigma_{22}=-\frac{q_{12} c_{11}-q_{11} c_{12}}{c_{11}} P_{3}^{2}+\frac{u_{m}}{s_{11}+s_{12}}, \\
& \sigma_{33}=-\frac{q_{11} c_{11}-q_{12} c_{12}}{c_{11}} P_{3}^{2}+\frac{u_{m}}{s_{11}+s_{12}}, \\
& u_{12}=u_{13}=u_{23}=0, u_{33}=u_{22}=u_{m}, \\
& u_{11}=\frac{q_{12}}{c_{11}} P_{3}^{2}-\frac{2 c_{12}}{c_{11}} u_{m}
\end{aligned}
$$

and the expansion coefficients renormalization

$a_{1}^{m}=a_{1}^{u}-\left(q_{11}+q_{12}\right) u_{m}+q_{12} \frac{2 c_{12}}{c_{11}} u_{m}, a_{11}^{m}=a_{11}^{u}-\frac{q_{12}^{2}}{c_{11}}$

It should be noted, that strain fields (23b) and (24b) satisfies compatibility conditions (20) for the case of onedimensional distribution of polarization in the form $P_{3}\left(x_{3}\right)$ and $P_{3}\left(x_{1}\right)$ respectively, since conditions (20) for onedimensional distributions is reduced to more simple forms, $\partial^{2} u_{11} / \partial x_{3}^{2}=\partial^{2} u_{22} / \partial x_{3}^{2}=0$ and $\partial^{2} u_{33} / \partial x_{1}^{2}=\partial^{2} u_{22} / \partial x_{1}^{2}=0$ respectively. The nontrivial stress components for different boundary conditions are summarized in Table 3 .

Table 3 Stress field in ferroelectric systems

\begin{tabular}{llll}
\hline & $\sigma_{11}$ & $\sigma_{22}$ & $\sigma_{33}$ \\
\hline $\begin{array}{l}\text { Free system } \\
\text { Clamped }\end{array}$ & 0 & 0 & 0 \\
$\quad-q_{12} P_{3}^{2}$ & $-q_{12} P_{3}^{2}$ & $-q_{11} P_{3}^{2}$ \\
$\begin{array}{c}\text { Films with } \\
\text { out of } \\
\text { plane } \mathbf{P}\end{array}$ & $\left(\frac{q_{11} c_{12}}{c_{11}}-q_{12}\right) P_{3}^{2}+\frac{u_{m}}{s_{11}+s_{12}}$ & 0 \\
$\begin{array}{c}\text { Films with } \\
\text { in plane } \mathbf{P}\end{array}$ & 0 & $\frac{q_{11} c_{12}-q_{12} c_{11}}{c_{11}} P_{3}^{2}+\frac{u_{m}}{s_{11}+s_{12}}$ & $\frac{q_{12} c_{12}-q_{11} c_{11}}{c_{11}} P_{3}^{2}+\frac{u_{m}}{s_{11}+s_{12}}$ \\
\hline
\end{tabular}


Appendix 2: the case of out of plane polarization with depolarization field present

Let us consider the case of film with out of plane polarization. The equation of state is

$\alpha P_{3}+\beta P_{3}^{3}-g \frac{\partial^{2} P_{3}}{\partial z^{2}}=E_{0}+E_{3}^{d}$,

with boundary conditions

$$
\left.\left(P_{3} \pm \lambda \frac{d P_{3}}{d z}\right)\right|_{z= \pm L / 2}=0 \text {, }
$$

For the considered 1D distribution of polarization the depolarization field can be written in the form

$E_{3}^{d}(z)=\frac{-P_{3}(z)+\bar{P}}{\varepsilon_{0} \varepsilon_{b}}-\frac{\bar{P}}{\varepsilon_{0} \varepsilon_{b}} \frac{H_{1} / \varepsilon_{g 1}+H_{2} / \varepsilon_{g 2}}{H_{1} / \varepsilon_{g 1}+H_{2} / \varepsilon_{g 2}+L / \varepsilon_{b}}$

Here $\varepsilon_{b}$ is the background permittivity of ferroelectric (see e.g. Refs. [1, 50, 52]), $\bar{P}=\int_{-L / 2}^{L / 2} P_{3}(z) d z / L$ is the averaged polarization, $\varepsilon_{g i}$ and $H_{i}$ for $i=1,2$ are respectively permittivity and thickness of dead layers between the film and its electrodes. It should be noted that similar expression was presented by Tilley [35] for the case of semiconductor electrodes with finite thickness, in this case $H_{i}$ should be considered as the values of screening radius and $\varepsilon_{g i}$ as the permittivity of electrodes.

The first term in Eq. 27 is similar to the expression for depolarization field obtained by Kretschmer and Binder [49] for the case of ideal electrodes and absence of background polarizability $\left(\varepsilon_{33}^{b} \equiv 1\right)$ and is determined by the polarization distribution. The second term in Eq. 27 is related to the non-ideal screening due to either dead layer or finite screening length. It should be noted, that only the latter term in Eq. 27 was considered by Tagantsev et al. [52].

Again, let us look for the solution of Eq. 25 in the form $P_{3}(z)=\bar{P}+p(z)$ with the deviation $p$ regarded small, $|p(z)| \ll|\bar{P}|$, and $\overline{p(z)} \equiv 0$. So, linearized problem (25)(26) acquires the form:

$\left\{\begin{array}{l}\left(\alpha+3 \beta \bar{P}^{2}+\frac{1}{\varepsilon_{0} \varepsilon_{b}}\right) p-g \frac{\partial^{2} p}{\partial z^{2}}=E_{0}-\left(\alpha \bar{P}+\beta \bar{P}^{3}\right)-\frac{\bar{P}}{\varepsilon_{0} \varepsilon_{b}} \Xi \\ \left.\left(p \pm \lambda \frac{d p}{d z}\right)\right|_{z= \pm L / 2}=-\bar{P}\end{array}\right.$

Here we introduced the following designation $\Xi \equiv$ $\frac{H_{1} / \varepsilon_{g 1}+H_{2} / \varepsilon_{g 2}}{H_{1} / \varepsilon_{g 1}+H_{2} / \varepsilon_{g 2}+L / \varepsilon_{b}}$.

The solution of the linear problem (28) has the form:

$P_{3}(z)=\frac{\varepsilon_{0} \varepsilon_{b}\left(E_{0}+2 \beta \bar{P}^{3}\right)+\bar{P}(1-\Xi)}{\varepsilon_{0} \varepsilon_{b}\left(\alpha+3 \beta \bar{P}^{2}\right)+1} f\left(z, L, R_{d}(\bar{P})\right)$, where the space distribution is governed by:

$f\left(z, L, R_{d}\right)=1-\frac{\cosh \left(z / R_{d}\right)}{\cosh \left(L / 2 R_{d}\right)+\left(\lambda / R_{d}\right) \sinh \left(L / 2 R_{d}\right)}$,

$R_{d}=\sqrt{\frac{\varepsilon_{0} \varepsilon_{b} g}{\varepsilon_{0} \varepsilon_{b}\left(\alpha+3 \beta \bar{P}^{2}\right)+1}}$.

The average polarization $\bar{P}$ should be determined selfconsistently from the spatial averaging of Eqs. 29a and 29b that leads to the following equation

$\bar{P}=\frac{\varepsilon_{0} \varepsilon_{b}\left(E_{0}+2 \beta \bar{P}^{3}\right)+\bar{P}(1-\Xi)}{\varepsilon_{0} \varepsilon_{b}\left(\alpha+3 \beta \bar{P}^{2}\right)+1} \bar{f}\left(L, R_{d}\right)$,

$\bar{f}\left(L, R_{d}\right)=1-\frac{\sinh \left(L /\left(2 R_{d}\right)\right) 2 R_{d} / L}{\cosh \left(L /\left(2 R_{d}\right)\right)+\left(\lambda / R_{d}\right) \sinh \left(L /\left(2 R_{d}\right)\right)}$.

Allowing for the dependence of characteristic length $R_{d}$ on average polarization, Eqs. $30 \mathrm{a}$ and $30 \mathrm{~b}$ is the transcendental equation for $\bar{P}$ determination. For the case of no external field, $E_{0}=0$, the equation (30a) could be rewritten in a more convenient way:

$\bar{P}=\sqrt{\frac{-\alpha-\frac{1-\Xi}{\varepsilon_{0} \varepsilon_{b}}\left(1-\bar{f}\left(L, R_{d}\right)\right)-\frac{\Xi}{\varepsilon_{0} \varepsilon_{b}}}{\beta\left(1+2\left(1-\bar{f}\left(L, R_{d}\right)\right)\right)}}$

However, in contrast to the case of in-plane polarization, the factor $\varepsilon_{0} \varepsilon_{b}$ drastically changes the situation (but it is not the case for ferromagnetic media). Since $\varepsilon_{0} \varepsilon_{b}\left(\alpha+3 \beta \bar{P}^{2}\right) \ll 1$ for the most of ferroelectrics, the following approximation is valid with high accuracy:

$R_{d}=\sqrt{\frac{\varepsilon_{0} \varepsilon_{b} g}{\varepsilon_{0} \varepsilon_{b}\left(\alpha+3 \beta \bar{P}^{2}\right)+1}} \approx R_{d 0}=\sqrt{\varepsilon_{0} \varepsilon_{b} g}=\mathrm{const}$

Using the approximation (31) Eq. 30c could be reduced to the following relation:

$\bar{P} \approx \sqrt{\frac{-\alpha-\frac{2 g}{\left(\lambda+R_{d 0}\right)} \frac{1}{\varepsilon_{b} \tilde{H}+L}-\frac{1}{\varepsilon_{0}} \frac{\tilde{H}}{\varepsilon_{b} \tilde{H}+L}}{\beta\left(1+\frac{4 \varepsilon_{0} \varepsilon_{b} g}{\left(\lambda+R_{d 0}\right) L}\right)}}$

Here $\tilde{H}=H_{1} / \varepsilon_{g 1}+H_{2} / \varepsilon_{g 2}$ is the effective thickness of dead layers (or effective screening radius of electrodes), responsible for imperfect screening. At $\tilde{H}=0 \mathrm{Eq} .32$ gives well known results for the ferroelectric films with out-ofplane polarization [47, 49]. In Eq. 32 we considered the limit $L \gg R_{d 0}$, which is valid for the most of films, since $R_{d 0}$ is of the order of lattice constant, and used relation $1-\bar{f}\left(L, R_{d 0}\right) \approx \frac{2 \varepsilon_{0} \varepsilon_{b} g}{\left(\lambda+R_{d 0}\right) L}$. 
It should be stressed that right hand side of Eq. 32 is independent on average polarization; in fact it is closed form solution of the problem. The numerator of this expression were obtained earlier by Glinchuk et al. [53] and Tagantsev et al. [52] in slightly different forms and using different methods. The consequences and the other physical properties of the considered system could be found in these references. Here we address only one issue, recently deserving especial attention, namely the value of depolarization field in the film center:

$$
\begin{aligned}
E_{3}^{d}(z=0) & =\frac{-P_{3}(z=0)+\bar{P}}{\varepsilon_{0} \varepsilon_{b}}-\frac{\bar{P}}{\varepsilon_{0} \varepsilon_{b}} \frac{\tilde{H}}{\tilde{H}+L / \varepsilon_{b}} \\
& \approx-\bar{P} \frac{2 g}{\left(\lambda+R_{d 0}\right) L}-\frac{\bar{P}}{\varepsilon_{0}} \frac{\tilde{H}}{\varepsilon_{b} \tilde{H}+L}
\end{aligned}
$$

Here we took into account that $\exp \left(-L / 2 R_{d}\right) \ll$ $1, f\left(z=0, L, R_{d}\right) \approx 1$ and Eq. 29 a could be rewritten as $P_{3}(z)=\bar{P} f\left(z, L, R_{d}\right) / \bar{f}\left(L, R_{d}\right)$. It is interesting to note, that Tagantsev et al. [52] dropped the first term (replacing $E_{3}^{d}(z=0)$ with average value $\left.\overline{E_{3}^{d}}\right)$ while considered the renormalization $-\alpha-\frac{2 g}{\left(\lambda+R_{d 0}\right)} \frac{1}{\varepsilon_{b} \tilde{H}+L}-\frac{1}{\varepsilon_{0}} \frac{\tilde{H}}{\varepsilon_{b} \tilde{H}+L}$ with both additives dependent on film thickness.

The exact but very cumbersome solution of the problem is also available in terms of elliptic integrals, while the numerical solution could be get by the phase field modeling (see e.g. [54]).

\section{Appendix 3: critical temperature dependence on the film thickness}

Expansion of left-hand side of Eq. 6a near the point $L \ll$ $R_{c 0}$ gives the following

$\frac{L}{2 R_{c 0}} \approx \frac{R_{c 0}}{\lambda}$

which is obviously valid for $\lambda \rightarrow \infty$.

Expansion near the pole of tan function $\left(L \sim \pi R_{c 0}\right)$ is (see e.g. [55])

$\frac{\left(L / R_{c 0}\right)}{(\pi / 2)^{2}-\left(L / 2 R_{c 0}\right)^{2}} \approx \frac{R_{c 0}}{\lambda}$.

which is relevant at $\lambda \rightarrow 0$.

Now the Eqs. 34a and 34b could be resolved via correlation radius and temperature and it allows one to derive the transition temperature in the explicit form. Namely, at $\lambda \rightarrow \infty$ :

$\alpha+\frac{2 g}{L \lambda}=0$

and at $\lambda \rightarrow 0$ : $\alpha+\frac{\pi^{2} g}{4 L \lambda+L^{2}}=0$

Pade approximation is

$\alpha+\frac{2 \pi^{2} g}{\pi^{2} L \lambda+2 L^{2}}=0$

\section{Appendix 4: polarization distribution in ferroelectric nanotubes}

In the case of the ferroelectric nanotubes the system (11) should be modified to take into account boundary condition on the inner surface of the tube as

$\left\{\begin{array}{l}\alpha P_{3}+\beta P_{3}^{3}-g\left(\frac{1}{\rho} \frac{\partial}{\partial \rho} \rho \frac{\partial}{\partial \rho}\right) P_{3}=E_{0} \\ \left.\left(P_{3}-\lambda_{1} \frac{d P_{3}}{d \rho}\right)\right|_{\rho=R_{1}}=\left.0 \quad\left(P_{3}+\lambda_{2} \frac{d P_{3}}{d \rho}\right)\right|_{\rho=R_{2}}=0\end{array}\right.$

As in previous sections we will look for the solution in the form $P_{3}(\rho)=\bar{P}+p(\rho)$, where $\bar{P}=2 \int_{R 1}^{R 2} P_{3}(\rho) \rho d \rho /$ $\left(R_{2}^{2}-R_{1}^{2}\right)$ is the averaged polarization, $p$ is the deviation, regarded small in ferroelectric phase: $|p(\rho)| \ll|\bar{P}|$. So, linearized problem (37) acquires the form:

$\left\{\begin{array}{l}\left(\alpha+3 \beta \bar{P}^{2}\right) p-g\left(\frac{\partial^{2}}{\partial \rho^{2}}+\frac{1}{\rho} \frac{\partial}{\partial \rho}\right) p=E_{0}-\left(\alpha \bar{P}+\beta \bar{P}^{3}\right), \\ \left.\left(p-\lambda_{1} \frac{\partial p}{\partial \rho}\right)\right|_{\rho=R_{1}}=-\bar{P},\left.\quad\left(p+\lambda_{2} \frac{\partial p}{\partial \rho}\right)\right|_{\rho=R_{2}}=-\bar{P}\end{array}\right.$

The solution of the problem (38) has the form:

$P_{3}(\rho)=\left(\frac{E_{0}+2 \beta \bar{P}^{3}}{\alpha+3 \beta \bar{P}^{2}}\right) t\left(\rho, R_{1}, R_{2}, R_{0}\right)$,

$$
\begin{aligned}
& t\left(\rho, R_{1}, R_{2}, R_{0}\right) \\
& =1+\frac{I_{0}\left(\frac{\rho}{R_{0}}\right)\left(K_{0}\left(\frac{R_{1}}{R_{0}}\right)-K_{0}\left(\frac{R_{2}}{R_{0}}\right)+\frac{\lambda_{1}}{R_{0}} K_{1}\left(\frac{R_{1}}{R_{0}}\right)+\frac{\lambda_{2}}{R_{0}} K_{1}\left(\frac{R_{2}}{R_{0}}\right)\right)}{\operatorname{Det}} \\
& +\frac{K_{0}\left(\frac{\rho}{R_{0}}\right)\left(-I_{0}\left(\frac{R_{1}}{R_{0}}\right)+I_{0}\left(\frac{R_{2}}{R_{0}}\right)+\frac{\lambda_{1}}{R_{0}} I_{1}\left(\frac{R_{1}}{R_{0}}\right)+\frac{\lambda_{2}}{R_{0}} I_{1}\left(\frac{R_{2}}{R_{0}}\right)\right)}{\operatorname{Det}},
\end{aligned}
$$

$$
\begin{aligned}
\text { Det }= & \left(I_{0}\left(\frac{R_{1}}{R_{0}}\right)-\frac{\lambda_{1}}{R_{0}} I_{1}\left(\frac{R_{1}}{R_{0}}\right)\right)\left(K_{0}\left(\frac{R_{2}}{R_{0}}\right)-\frac{\lambda_{2}}{R_{0}} K_{2}\left(\frac{R_{2}}{R_{0}}\right)\right) \\
& -\left(I_{0}\left(\frac{R_{2}}{R_{0}}\right)+\frac{\lambda_{2}}{R_{0}} I_{1}\left(\frac{R_{2}}{R_{0}}\right)\right)\left(K_{0}\left(\frac{R_{1}}{R_{0}}\right)+\frac{\lambda_{1}}{R_{0}} K_{1}\left(\frac{R_{1}}{R_{0}}\right)\right)
\end{aligned}
$$

The equation for the average polarization could be found after the averaging of (39a) in the following form

$\bar{P}=\left(\frac{E_{0}+2 \beta \bar{P}^{3}}{\alpha+3 \beta \bar{P}^{2}}\right) \bar{t}\left(R_{1}, R_{2}, R_{0}\right)$, 


$$
\begin{aligned}
\bar{t}\left(R_{1}, R_{2}, R_{0}\right)= & 1 \\
& +\frac{R_{0}\left(R_{2} I_{1}\left(\frac{R_{2}}{R_{0}}\right)-R_{1} I_{1}\left(\frac{R_{1}}{R_{0}}\right)\right)\left(K_{0}\left(\frac{R_{1}}{R_{0}}\right)-K_{0}\left(\frac{R_{2}}{R_{0}}\right)+\frac{\lambda_{1}}{R_{0}} K_{1}\left(\frac{R_{1}}{R_{0}}\right)+\frac{\lambda_{2}}{R_{0}} K_{1}\left(\frac{R_{2}}{R_{0}}\right)\right)}{\operatorname{Det}\left(R_{2}^{2}-R_{1}^{2}\right) / 2} \\
& +\frac{R_{0}\left(-R_{2} K_{1}\left(\frac{R_{2}}{R_{0}}\right)+R_{1} K_{1}\left(\frac{R_{1}}{R_{0}}\right)\right)\left(-I_{0}\left(\frac{R_{1}}{R_{0}}\right)+I_{0}\left(\frac{R_{2}}{R_{0}}\right)+\frac{\lambda_{1}}{R_{0}} I_{1}\left(\frac{R_{1}}{R_{0}}\right)+\frac{\lambda_{2}}{R_{0}} I_{1}\left(\frac{R_{2}}{R_{0}}\right)\right)}{\operatorname{Det}\left(R_{2}^{2}-R_{1}^{2}\right) / 2} .
\end{aligned}
$$

Open Access This article is distributed under the terms of the Creative Commons Attribution Noncommercial License which permits any noncommercial use, distribution, and reproduction in any medium, provided the original author(s) and source are credited.

\section{References}

1. Lines ME, Glass AM (1977) Principles and applications of ferroelectrics and related phenomena. Clarendon Press, Oxford

2. Wadhawan VK (2000) Introduction to ferroic materials. Gordon and Breach Science Publishers, New York

3. Scott JF (2007) Nat Mater 6:256

4. Fong DD, Stephenson GB, Streiffer SK, Eastman JA, Auciello O, Fuoss PH, Thompson C (2004) Science 304:1650

5. Erdem E, Semmelhack H-C, Bottcher R, Rumpf H, Banys J, Matthes A, Glasel H-J, Hirsch D, Hartmann E (2006) J Phys Condens Matter 18:3861

6. Luo Y, Szafraniak I, Zakharov ND, Nagarajan V, Steinhart M, Wehrspohn RB, Wendorff JH, Ramesh R, Alexe M (2003) Appl Phys Lett 83:440

7. Morrison FD, Ramsay L, Scott JF (2003) J Phys Condens Matter 15:L527

8. Mishina ED, Vorotilov KA, Vasil'ev VA, Sigov AS, Ohta N, Nakabayashi S (2002) J Exp Theor Phys 95:502

9. Nourmohammadi A, Bahrevar MA, Schulze S, Hietschold M (2008) J Mater Sci 43:4753. doi:10.1007/s10853-008-2665-3

10. Yadlovker D, Berger S (2005) Phys Rev B 71:184112-1-6

11. Zhou ZH, Gao XS, Wang J, Fujihara K, Ramakrishna S, Nagarajan V (2007) Appl Phys Lett 90:052902-1-3

12. Poyato R, Huey BD, Padture NP (2006) J Mater Res 21:547

13. Gruverman A, Kalinin SV (2006) J Mater Sci 41:107. doi: 10.1007/s10853-005-5946-0

14. Sidorkin AS (2006) Domain structure in ferroelectrics and related materials. Cambridge international science publishing, Cambridge

15. Ghosez P, Junquera J (2006) First-principles modeling of ferroelectric oxides nanostructures. In: Rieth M, Schommers W (eds) Handbook of theoretical and computational nanotechnology (Chap 134). American Scientifc Publisher, Stevenson Ranch

16. Geneste G, Bousquest E, Junquera J, Chosez P (2006) Appl Phys Lett 88:112906-1-3

17. Morozovska AN, Glinchuk MD, Eliseev EA (2007) Phys Rev B 76:014102-1-13

18. Kornev IA, Fu H, Bellaiche L (2006) J Mater Sci 41:137. doi: 10.1007/s10853-005-5962-0

19. Aguado-Puente P, Junquera J (2008) Phys Rev Lett 100:177601$1-4$
20. Huang H, Sun CQ, Zh Tianshu, Hing P (2001) Phys Rev B 63:184112-1-9

21. Glinchuk MD, Morozovska AN (2003) Phys State Sol (B) 238:81

22. Akdogan EK, Safari A (2007) J Appl Phys 101:064114-1-8

23. Catalan G, Sinnamon LJ, Gregg JM (2004) J Phys Condens Matter 16:2253

24. Catalan G, Noheda B, McAneney J, Sinnamon LJ, Gregg JM (2005) Phys Rev B 72:020102(R)-1-4

25. Majdoub MS, Sharma P, Cagin T (2008) Phys Rev B 77:1254241-9

26. Majdoub MS, Sharma P, Cagin T (2008) Phys Rev B 78: 121407(R)-1-4

27. Kalinin SV, Meunier V (2008) Phys Rev B 77:033403-1-4

28. Ban Z-G, Alpay SP, Mantese JV (2003) Phys Rev B 67:184104$1-6$

29. Akcay G, Alpay SP, Rossetti GA, Scott JF (2008) J Appl Phys 103:024104-1-7

30. Qiu QY, Nagarajan V, Alpay SP (2008) Phys Rev B 78:064117$1-13$

31. Roduner E (2006) NanoscopicMaterials. Size-dependent phenomena. Royal Society of Chemistry Publishing, Cambridge

32. Wang CL, Smith SRP (1995) J Phys Condens Matter 7:7163

33. Rychetsky I, Hudak O (1997) J Phys Condens Matter 9:4955

34. Landau LD, Lifshitz EM (1980) Electrodynamics of continuous media. Butterworth Heinemann, Oxford

35. Tilley DR (1996) In: Paz de Araujo C, Scott JF, Teylor GW (eds) Ferroelectric thin films. Gordon and Breach, Amsterdam, pp 11-45

36. Bratkovsky AM, Levanyuk AP (2005) Phys Rev Lett 94:107601$1-4$

37. Glinchuk MD, Eliseev EA, Stephanovich VA, Farhi R (2003) J Appl Phys 93:1150

38. Morozovska AN, Eliseev EA, Glinchuk MD (2006) Phys Rev B 73:214106-1-13

39. Morozovska AN, Eliseev EA, Glinchuk MD (2007) Physica B $387: 358$

40. Timoshenko SP, Goodier JN (1970) Theory of elasticity. McGraw-Hill, New-York

41. Pertsev NA, Zembilgotov AG, Tagantsev AK (1998) Phys Rev Lett 80:1988

42. Cao W, Cross LE (1991) Phys Rev B 44:5

43. Zhirnov VA (1959) Zh eksp theor Fiz 35:1175 [(1959) Sov Phys JETP 8:822-827]

44. Speck JS, Pompe W (1994) Theory J Appl Phys 76:466

45. Tilley DR, Zeks B (1984) Solid State Commun 49:823

46. Ong L-H, Osman J, Tilley DR (2001) Phys Rev B 63:144109-110

47. Glinchuk MD, Eliseev EA, Stephanovich VA (2002) Phys B Condens Matter 322:356

48. Gradshteyn IS, Ryzhik IM (1994) In: Jeffrey A (ed) Table of integrals, series, and products, 5th edn. Academic, New York 
49. Kretschmer R, Binder K (1979) Phys Rev B 20:1065

50. Woo CH, Zheng Y (2008) Appl Phys A: Mater Sci Process 91:59

51. Morozovska AN, Eliseev EA, Glinchuk MD, Blinc R (2008) Phys Rev B (accepted)

52. Tagantsev AK, Gerra G, Setter N (2008) Phys Rev B 77:174111$1-9$
53. Glinchuk MD, Zaulychny BY, Stephanovich VA (2006) Physica Status Solidi (B) 243:542

54. Chen L-Q (2008) J Am Ceram Soc 91:1835

55. Korn GA, Korn TM (1961) Mathematical handbook for scientists and engineers. McGraw-Hill, New-York 